\title{
Fitness Attributes of Fusarium graminearum Isolates from Wheat in New York Possessing a 3-ADON or 15-ADON Trichothecene Genotype
}

\author{
Pierri Spolti, Emerson M. Del Ponte, Jaime A. Cummings, Yanhong Dong, and Gary C. Bergstrom
}

First, third, and fifth authors: Department of Plant Pathology and Plant-Microbe Biology, Cornell University, Ithaca, NY 14853-5904; first and second authors: Departamento de Fitossanidade, Universidade Federal do Rio Grande do Sul, Porto Alegre, RS 91540000 Brazil; and fourth author: Department of Plant Pathology, University of Minnesota, St. Paul 55108.

Accepted for publication 10 November 2013.

\begin{abstract}
Spolti, P., Del Ponte, E. M., Cummings, J. A., Dong, Y., and Bergstrom, G. C. 2014. Fitness attributes of Fusarium graminearum isolates from wheat in New York possessing a 3-ADON or 15-ADON trichothecene genotype. Phytopathology 104:513-519.

In all, 50 isolates of Fusarium graminearum from wheat spikes in New York, including 25 isolates each of the 15-acetyl-deoxynivalenol (15-ADON) and 3-ADON genotype, were tested to determine whether 3-ADON isolates are more fit for saprophytic survival and pathogenicity on wheat spikes than are $15-\mathrm{ADON}$ isolates. The isolates were characterized and compared for 14 different attributes of saprophytic fitness and pathogenic fitness on a susceptible wheat variety. Isolates of the two genotypes could

not be differentiated for most of these traits. Three principle components - ascospore production on corn stalks, total trichothecene amount in wheat kernels, and incidence of diseased spikelets up from the point of inoculation-accounted for $29.4,18.9$, and $10.8 \%$ of the variation among the isolates, respectively. A bootstrapping procedure grouped the isolates into two distinct groups, with 27 and 23 isolates each, with isolates from both genotypes represented in similar proportions (15-ADON/3-ADON, $n=14 / 13$ and 11/12). Within the contemporary population of F. graminearum causing wheat head blight in New York, isolates with a 3-ADON genotype did not possess any detectable advantage over isolates with a 15-ADON genotype in saprophytic fitness or in pathogenic fitness on a susceptible wheat cultivar.
\end{abstract}

Fusarium head blight (FHB) is one of the most devastating diseases of wheat worldwide and is of increasing concern in North America due to the economic losses caused by yield reduction and rejection of grain contaminated with the mycotoxin deoxynivalenol (DON) $(23,24,31)$. The disease is mainly caused by Fusarium graminearum sensu lato, which comprises several biogeographically structured and phylogenetically distinct species $(46,49)$. In North America, F. graminearum sensu stricto (hereafter $F$. graminearum) is the dominant species associated with FHB epidemics in wheat $(23,31,47,57)$.

Isolates of $F$. graminearum produce a range of mycotoxins but the most significant are those of the B-trichothecene class such as DON, nivalenol (NIV), and the DON-acetylated forms. Chemical analysis of inoculated artificial substrates or host tissues is used to assign a particular isolate to a specific chemotype based on the mycotoxin produced in greatest quantity (12).

Polymerase chain reaction (PCR) primers targeting portions of genes related to trichothecene synthesis have been developed to genotype isolates as predictive but not absolutely definitive of the trichothecene chemotype (i.e., chemical phenotype), most commonly to determine the 3-acetyl-deoxynivalenol (3-ADON), 15ADON, or NIV genotype $(6,34,57)$. Thus, molecular surveillance is revealing the distribution of the three trichothecene genotypes in populations of $F$. graminearum sensu lato around the world $(3,4,25,34,36,45,47,49,57)$. Both the DON and the NIV genotypes have been found in all surveyed regions and, between the two acetylates of DON, 15-ADON appears to be dominant in North America $(4,47,57)$, South America $(3,36,45)$, and Europe $(34,35)$.

Corresponding author: G. C. Bergstrom; E-mail address: gcb3@ cornell.edu

* The $e$-Xtra logo stands for "electronic extra" and indicates that the online version contains one supplemental Figure.

http://dx.doi.org/10.1094/PHYTO-07-13-0206-R

(C) 2014 The American Phytopathological Society
An apparent temporal or spatial shift from the 15-ADON to the 3-ADON genotype has been suggested based on an increased prevalence of 3-ADON in contemporary compared with historical populations from Minnesota and eastern Canada $(4,57)$. A series of studies was conducted to understand potential factors leading to the shift such as measures of pathogenic traits which would provide advantages for 3-ADON compared with 15-ADON isolates $(14,20,44,55,57)$.

Some studies showed that $3-A D O N$ isolates were able to (i) produce higher amounts of DON during pathogenesis $(44,57)$, (ii) grow faster on artificial medium (57), and (iii) be more aggressive on wheat $(44,55,57)$ than 15 -ADON isolates. Conversely, no difference in aggressiveness on wheat and barley lines was detected between antecedent (15-ADON) and emerging (3-ADON) populations from the Midwestern United States, although the latter tended to produce higher amounts of DON (20). Likewise, measures of aggressiveness on wheat (FHB severity, Fusariumdamaged kernels [FDK], and DON production) did not discriminate the $3-\mathrm{ADON}$ producers and the emergent $15-\mathrm{ADON}$ populations from eastern Canada (four isolates were characterized per population) (17).

All of these previous studies have focused mainly on the characterization of pathogenic traits $(14,17,20,44,55,57)$. In spite of the key role played by the saprophytic stage of $F$. graminearum in survival between crop seasons and as a source of inoculum for FHB epidemics $(1,13,27,29,50,54)$, only two studies $(44,57)$ investigated differences in mycelial growth rate, as a measure of saprophytic fitness, and showed conflicting results that 3-ADON isolates had higher mycelial growth rate than $15-\mathrm{ADON}$ isolates (57) or no significant differences were observed between these two genotype groups (44).

We utilized 15-ADON and 3-ADON isolates from contemporary populations of $F$. graminearum in New York to test the dual hypotheses that 3-ADON isolates are more fit as saprophytes and as pathogens of wheat than their 15-ADON counterparts. We 
considered fungal overall fitness in regard to several traits of saprophytic survival, sexual reproduction, mycotoxin production, and pathogenicity on wheat spikes. An abstract containing preliminary findings from this study was published (48).

\section{MATERIALS AND METHODS}

Fungal isolates. The $50 \mathrm{~F}$. graminearum isolates used in this study were selected from a large collection of isolates obtained from wheat spikes with FHB symptoms in a survey of commercial fields in western New York in 2011 (G. C. Bergstrom, unpublished data). Isolates in the large collection were categorized for B-trichothecene genotypes based on PCR assays targeting Tri3 and Tri12 genes (47,56). The Tri3 and Tri12 genes have been utilized to identify trichothecene genotypes corresponding to putative chemotypes (3-ADON genotype, 15-ADON genotype, and NIV genotype) where, for each primer set, a PCR amplicon of characteristic size is associated with each of the trichothecene genotypes (31). Trichothecene genotypes were recovered in the ratio of $15 \% 3-\mathrm{ADON} / 85 \% 15-\mathrm{ADON}$, and no isolates of the NIV genotype were recovered (G. C. Bergstrom, unpublished data). Twenty-five isolates each of the 3-ADON and 15-ADON genotypes were selected arbitrarily from nine commercial wheat fields in 2011 (Fig. 1).

Mycelial growth rate and temperature sensitivity. Spore suspensions $(1,000$ spores $/ \mathrm{ml})$ were obtained from 5-day-old cultures grown on quarter-strength potato dextrose agar (PDA) under permanent black light (type F40/350BL; Sylvania, Danvers MA). On a 9-cm PDA plate, three equidistant colonies were formed by dropping $5 \mu \mathrm{l}$ of the suspension following the procedures proposed by Zhan and McDonald (58): (i) all media were prepared as a single batch by a single person during a single day, (ii) a machine was used to dispense the same volume $(7 \mathrm{ml})$ of PDA into each petri dish, (iii) inoculated plates were kept stationary on a bench for $5 \mathrm{~min}$ before being placed at random in the incubators, and (iv) the entire inoculation procedure was conducted by one person during the course of a single day. The cultures were incubated at each of four temperatures $\left(15,20,25\right.$, or $\left.30^{\circ} \mathrm{C}\right)$ in darkness for 5 days. The colony diameter was measured from two perpendicular directions and mean radial growth rate (millimeters per day) was determined. Mycelia growth rates were compared at $25^{\circ} \mathrm{C}$ (57). Sensitivity to temperature for each isolate was obtained by the ratio between isolate growth rate at $30^{\circ} \mathrm{C}$ and its respective rate at $15^{\circ} \mathrm{C}$ (58). Three replicates (incubator) were used for each combination of isolate (one petri dish per isolate) and temperature.
Perithecia formation and ascospore production on carrot agar. Perithecia formation and ascospore production were assessed following a standard protocol (5). Briefly, carrot agar (28) in a 6.0-cm-diameter petri dish was inoculated in the center with each of the $50 \mathrm{~F}$. graminearum isolates. Each petri dish was placed under bright fluorescent lights (type C-14; Underwriters Laboratories Inc., Northbrook, IL) at room temperature $\left(23^{\circ} \mathrm{C}\right)$ and the isolate was allowed to grow until mycelium had reached the outer edge of the petri dish ( 5 days). The aerial mycelia were then removed with a sterile toothpick and an aliquot of $1 \mathrm{ml}$ of $2.5 \%$ Tween 60 was added to the surface. The formation of perithecia was visually checked $(5,7)$ at 3 and 6 days after returning the plates to light. Perithecia formation was scored as - (no perithecia formed) or,+++ , and +++ when the perithecia covered 1 to 10 , 11 to 30 , and $>30 \%$ of the petri dish area, respectively. At 6 days after application of Tween solution, six 1-cm-diameter plugs were cut out of the agar with a cork borer from each plate. Each plug was sliced in half across the center of the circle and three halfplugs were placed on a glass microscope slide. The slides were placed in a humidity chamber overnight under lights and the accumulated spores washed off from the slide with water and quantified using a hemacytometer. There were four replicated humid chambers with 50 slides each (one slide per isolate).

Perithecia formation and ascospore discharge on corn stalks. Corn stalk segments (half-cylinders $4 \mathrm{~cm}$ long by $2.5 \mathrm{~cm}$ wide) were soaked in deionized distilled water overnight $(10 \mathrm{~h})$ and autoclaved on three consecutive days. Each sterilized stalk segment was placed with the flat pith surface facing down and the convex rind surface facing up, then inoculated by placing on the rind surface 15 discs ( $5 \mathrm{~mm}$ in diameter and $2 \mathrm{~mm}$ deep) of PDA colonized by one of the 50 isolates. Inoculated segments (two per isolate per replicate) were incubated under moist condition (relative humidity near $100 \%)$ at room temperature $\left(\approx 22^{\circ} \mathrm{C}\right)$. At 1 week after inoculation, the PDA discs were consumed by fungus growth and barely visible as a film. At 21 days after inoculation, the stalks were incubated for two additional days under continuous fluorescent light (type C-14; Underwriters Laboratories Inc.) for ascospore release (30). The number of discharged ascospores was estimated based on the number of CFU counted on water-agar plates that were positioned $1.5 \mathrm{~cm}$ above the two segments during the 2-day period. Four replicates (humid chambers) with 100 corn stalk segments (2 segments per isolate per replicate) were used. The capacity to form perithecia was not subjected to a statistical analysis but was used as a descriptive characteristic (7) of the two genotypes on both carrot agar and inoculated corn stalks.

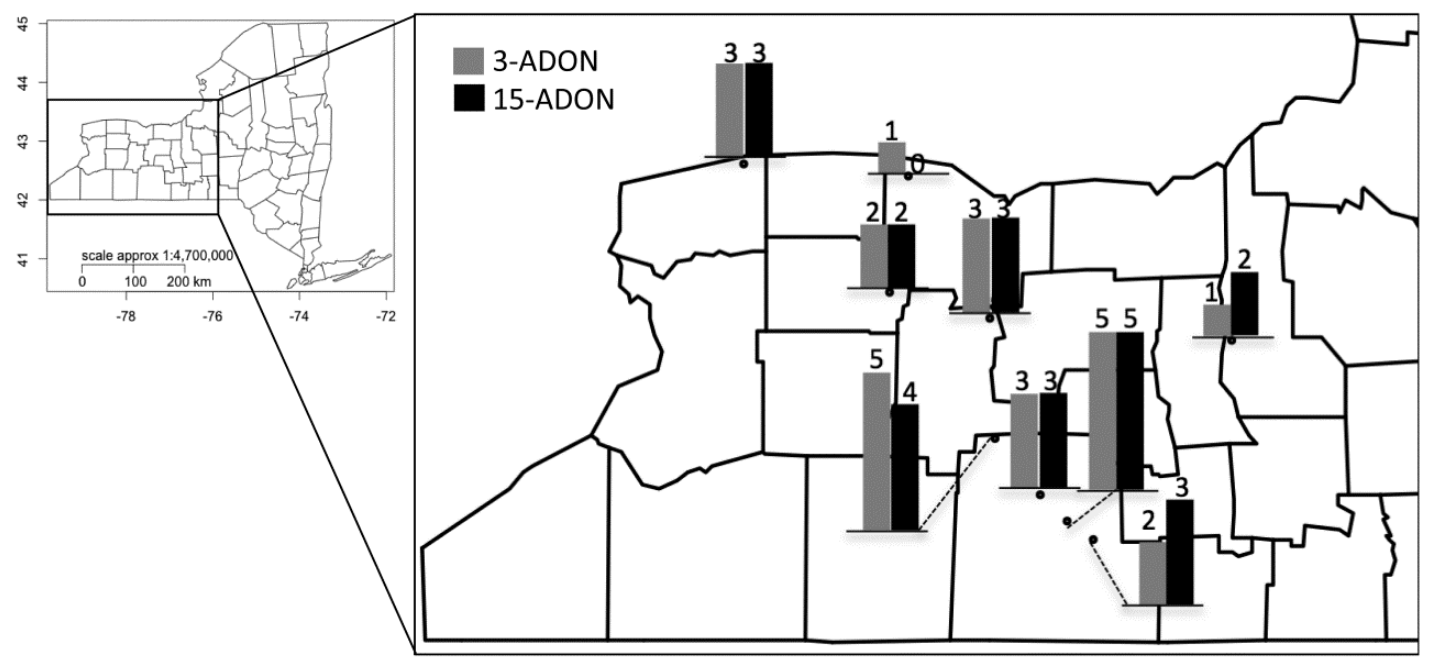

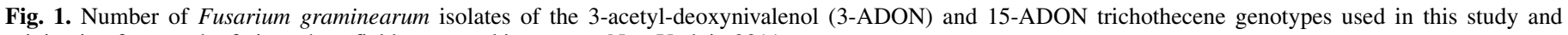
originating from each of nine wheat fields surveyed in western New York in 2011. 
Aggressiveness on a susceptible wheat variety. Seed of 'Norm', a spring wheat variety highly susceptible to FHB, were sown in $15.3-\mathrm{cm}$ diameter clay pots (10 seeds/pot) filled with soilless Cornell Mix (containing approximately 97 liters of compressed peat moss, 114 liters of vermiculite, $9.1 \mathrm{~kg}$ of dolomitic limestone, and $2.7 \mathrm{~kg}$ of 11-5-11 fertilizer). Plants were grown for 8 weeks in the greenhouse at 20 to $25^{\circ} \mathrm{C}$ under 12 to $14 \mathrm{~h}$ of light per day and trimmed to keep the 10 main tillers in each pot. Potted plants were maintained in the greenhouse from planting to inoculation (10). Wheat heads were inoculated at early milk stage (Zadok's 73), previously determined as the most susceptible stage in Norm (11). The central spikelet of each spike was marked and a $20-\mu \mathrm{l}$ drop of the spore suspension $\left(5 \times 10^{4}\right.$ macroconidia/ml $)$ was placed inside the spikelet. Pots were individually covered with a plastic bag and kept for $48 \mathrm{~h}$ in a growth chamber set at $25.5^{\circ} \mathrm{C}$ and a cycle of 14 and $9 \mathrm{~h}$ of light and darkness, respectively. The bags were then removed and the plants remained in the same chamber until harvest $(\approx 45$ days after inoculation). Aggressiveness of each isolate was determined based on assessments of the percentage of diseased spikelets (both for the whole spike and for spikelets above or below the central spikelet) per inoculated spike made at 10 days after inoculation. Because only one pot was used per isolate, the 10 spikes were considered pseudoreplicates. Mycotoxin production by each of the 50 isolates was assessed from the bulk of wheat kernels from all inoculated spikes. Prior to analysis, the kernels were ground and a 5-g sample of each replicate was obtained. The amount of DON and its acetylated forms (15-ADON and 3-ADON) were quantified using a gas chromatography-mass spectrometry method (18,33).

Data analysis. For all experiments, except the aggressiveness test, a completely randomized design was used to analyze the data; the experiments were performed twice and data combined for analysis. For the univariate analysis, all the variables listed in Table 1 were submitted to variance analysis via permutation test (41). The significance of the permutation test was evaluated by comparing the observed test statistic with the distribution of 10,000 random test statistics. Statistical significance was considered at the $P<0.05$ level, comparing the trichothecene genotypes. For

TABLE 1. Saprophytic traits of 50 Fusarium graminearum isolates causing Fusarium head blight of wheat in New York in 2011, and possessing either a 3-acetyl-deoxynivalenol (3-ADON) or a 15-ADON trichothecene genotype

\begin{tabular}{|c|c|c|c|}
\hline \multirow[b]{2}{*}{ Variable } & \multicolumn{2}{|c|}{ Trichothecene genotype ${ }^{a}$} & \multirow[b]{2}{*}{$P^{\mathrm{b}}$} \\
\hline & $15-\mathrm{ADON}$ & 3-ADON & \\
\hline \multicolumn{4}{|l|}{ Temperature $\left({ }^{\circ} \mathrm{C}\right)^{\mathrm{c}}$} \\
\hline 15 & $3.25 \pm 1.26$ & $2.61 \pm 1.50$ & 0.345 \\
\hline 20 & $8.76 \pm 2.45$ & $9.30 \pm 1.93$ & 0.471 \\
\hline 25 & $11.75 \pm 1.86$ & $11.73 \pm 1.87$ & 0.573 \\
\hline 30 & $9.37 \pm 4.05$ & $10.53 \pm 3.44$ & 0.203 \\
\hline Sensitivityd & $3.27 \pm 1.42$ & $3.75 \pm 1.24$ & 0.065 \\
\hline \multicolumn{4}{|l|}{ Ascospores discharged ${ }^{\mathrm{e}}$} \\
\hline CA $\left(\text { ascospores } / \mathrm{mm}^{2}\right)^{\mathrm{f}}$ & $187.70 \pm 122.90$ & $439.40 \pm 438.30$ & 0.041 \\
\hline $\begin{array}{l}\text { Corn stalk } \\
\text { (CFU/plate) }\end{array}$ & $36.10 \pm 34.40$ & $41.30 \pm 30.80$ & 0.729 \\
\hline
\end{tabular}

${ }^{a}$ Isolates were identified to trichothecene genotype using polymerase chain reaction assays targeting Tri3 and Tril2 genes (56). In total, 25 15-ADON and 253 -ADON isolates were used in this study. Data shown are means \pm standard deviation.

${ }^{b}$ Significant difference comparing trichothecene genotypes was estimated by permutation test.

${ }^{\mathrm{c}}$ Mycelial growth determined on potato dextrose agar (PDA) at the fifth day of incubation.

${ }^{d}$ Temperature sensitivity; the ratio of the mycelial growth rate at 30 and $15^{\circ} \mathrm{C}$ on PDA after 5 days of incubation.

${ }^{\mathrm{e}}$ Ascospores produced from carrot agar (CA) or from sterilized and inoculated corn stalk.

${ }^{\mathrm{f}}$ Counting ascospores from CA media.

$\mathrm{g}$ Counting of CFU on agar media resulting from discharge of ascospores from inoculated corn stalks. the aggressiveness assay on Norm wheat, the confidence interval $(95 \%)$ was used to allow determination of the statistical difference between the two genotypic groups. For correlation analysis (Spearman), principal component analysis (PCA), and cluster analyses, all the data on isolate characterization listed in Table 1 (the mycelial growth rate was estimated at $25^{\circ} \mathrm{C}$ ) and Table 2 (plus the severity of FHB above or below the central floret) were averaged over replicates to form a mean value for each isolate, and each column was considered as a separate variable. This created a data matrix with 50 isolates (rows) and 14 variables (columns). Cluster and PCA were subjected to bootstrap analysis (10,000 permutations) to verify cluster group sharpness and ordination axis stability $(38,39)$. In the cluster analysis, incremental sum of squares was used as the clustering criterion based on Gower index resemblance measure, assuming the cluster groups sharpness when $P \geq 0.05$. All analyses were performed with MULTIV software version 2.4 (40).

\section{RESULTS}

Saprophytic traits. The mean of the mycelial growth rates did not differ significantly between the two genotypes compared at all tested temperatures $(P \geq 0.203)$ and the 3 -ADON isolates were not more sensitive to temperature than the 15 -ADON isolates $(P=$ 0.065 ) (Table 1). Regardless of the substrate, there was considerable variation among the isolates within each genotype in their capacity to form perithecia. Only two isolates (NY600 and NY693) did not form perithecia during colonization of the two substrates. In general, the 15-ADON isolates were faster and more abundant producers of perithecia on carrot agar medium than the 3-ADON isolates. However, such difference was not observed for the colonization of corn stalks, in which relatively fewer perithecia were formed than on carrot agar (Fig. 2). Though they formed perithecia slowly on carrot agar, the 3-ADON isolates produced $2 \times$ more ascospores than the 15 -ADON isolates on carrot agar $(P=0.041)$. Such differences were not observed in the mean number of colonies arising from spores released from perithecia formed on corn stalks $(P=0.729)$ (Table 1).

TABLE 2. Pathogenic traits of 50 Fusarium graminearum isolates causing Fusarium head blight (FHB) of wheat in New York in 2011, and possessing either a 3-acetyl-deoxynivalenol (3-DON) or a 15-ADON trichothecene genotype

\begin{tabular}{|c|c|c|}
\hline \multirow[b]{2}{*}{ Variables $^{\mathrm{a}}$} & \multicolumn{2}{|c|}{ Trichothecene genotype ${ }^{b}$} \\
\hline & $15-\mathrm{ADON}$ & 3-ADON \\
\hline Severity $(\%)^{\mathrm{c}}$ & $39.32 \pm 9.10$ & $40.41 \pm 9.60$ \\
\hline Fusarium-damaged kernels $(\%)^{\mathrm{d}}$ & $54.71 \pm 15.04$ & $53.90 \pm 13.82$ \\
\hline Thousand-kernel weight (g) & $14.02 \pm 1.8$ & $13.00 \pm 1.63$ \\
\hline Relative weight damage $(\%)^{\mathrm{e}}$ & $47.41 \pm 7.04$ & $47.20 \pm 5.97$ \\
\hline Deoxynivalenol $(\mu \mathrm{g} / \mathrm{g})^{\mathrm{f}}$ & $41.43 \pm 15.72$ & $71.10 \pm 26.93$ \\
\hline $15-\operatorname{ADON}(\mu \mathrm{g} / \mathrm{g})^{\mathrm{f}}$ & $0.60 \pm 0.30$ & $0.20 \pm 0.10$ \\
\hline $3-\mathrm{ADON}(\mu \mathrm{g} / \mathrm{g})^{\mathrm{f}}$ & $0.50 \pm 0.20$ & $1.20 \pm 0.40$ \\
\hline Total trichothecene $(\mu \mathrm{g} / \mathrm{g})^{\mathrm{g}}$ & $42.70 \pm 16.20$ & $73.10 \pm 27.40$ \\
\hline
\end{tabular}

a FHB intensity on 'Norm' plants inoculated by the central floret method.

${ }^{\mathrm{b}}$ Isolates were identified to trichothecene genotype using polymerase chain reaction assays targeting Tri3 and Tri12 genes (56). In total, 25 15-ADON and 253 -ADON isolates were used in this study. Data shown are means \pm confidence interval $(95 \%)$

c Percentage of diseased spikelets in inoculated spikes at 10 days after inoculation of the central spikelet.

d Fusarium-damaged kernels visually estimated in the total of kernels per isolate.

e Relative difference in kernel weight between kernels from noninoculated and inoculated plants.

$\mathrm{f}$ Trichothecene amount in grains from spikes of Norm wheat inoculated in the greenhouse and averaged from 10 heads; ppm = part per million $(\mu \mathrm{g} / \mathrm{g}$ of samples).

$\mathrm{g}$ Total amount of trichothecenes (DON + 15-ADON + 3-ADON) in grains from spikes of Norm wheat inoculated in the greenhouse. 
Aggressiveness and trichothecene production. All isolates successfully infected the susceptible wheat variety but the levels of aggressiveness, measured by two disease variables (FHB severity and FDK) and two yield-related variables (thousand-kernel weight and relative weight damage), varied considerably and significantly among the isolates of the same genotype group, which contributed to the lack of difference between the two groups of trichothecene genotypes (Table 2). Isolates exhibiting a similarly low or high aggressiveness were observed in each genotype group (Fig. 3; Supplemental Figure 1). A higher proportion of diseased spikelets was found below the inoculation point $(P=0.023)$, regardless of the genotype $(P=0.928)$. All isolates produced DON in mature kernels at levels of 0.25 to $238.30 \mu \mathrm{g} / \mathrm{g}$ (Fig. 3). DON levels were much higher than those of the two acetylates (Table 2), in which the maximum level of $3.4 \mu \mathrm{g} / \mathrm{g}$ was found for a 3-ADON isolate (Fig. 4). Because of the large variance in DON levels for isolates of the same trichothecene genotype, no difference was found for the mean of DON between the two trichothecene genotypes (Table 2). In all, 10 and 6 isolates of 15ADON and 3-ADON, respectively, did not produce detectable levels of at least one acetylated form of DON. The 15-ADON isolates produced a higher amount of this acetylate form than did 3-ADON isolates. The same pattern was observed for 3-ADON isolates that produced more of this form than 15-ADON isolates (Table 2). No difference was found between the two trichothecene genotypes for the total mean of DON plus its acetylated forms (15-ADON + 3-ADON) (Table 2).

For the 15-ADON group, 15 isolates also produced detectable levels of 3-ADON. Two 15-ADON isolates produced 3-ADON and not $15-\mathrm{ADON}$, and one $15-\mathrm{ADON}$ isolate produced $2 \times$ more 3 -ADON than 15-ADON (Fig. 4). For the 3-ADON group, only four isolates produced detectable levels of 15-ADON. All but two 3-ADON isolates produced 3-ADON more abundantly than 15ADON. Thus, it appears that PCR-derived genotype did not accurately predict the chemotype for 5 of the 50 isolates. Three of the 15-ADON genotypes were actually 3-ADON chemotypes and two 3-ADON genotypes were actually 15 -ADON chemotypes. In light of this, we reanalyzed the data grouped by isolates of each chemotype group (26 3-ADON versus 24 15-ADON); statistical inferences about chemotype groups did not differ from those made for comparison of molecular genotype groups (25 3-ADON and 25 15-ADON). Therefore, the trichothecene genotypes are retained in the figures and tables.
Overall fitness of the trichothecene genotypes. Correlation analysis for each pairwise comparison $(n=91)$ for all 14 variables used to characterize saprophytic and pathogenic fitness of the two trichothecene groups (Tables 1 and 2) showed 49 significant $(P<$ $0.05)$ correlations. Among the saprophytic traits, mycelial growth rate was positively correlated with ascospores produced on corn stalks $(P=0.01)$ but not on carrot agar $(P=0.21)$. Highest correlation values were found between measures describing pathogenic traits, especially disease severity that was highly correlated with yield-related variables and total trichothecene levels $(P<$ 0.01) (data not shown).

In the PCA, ascospores produced on corn stalks, total amount of trichothecenes in wheat kernels, and FHB severity in spikelets up from the point of inoculation showed the highest correlations with the first, second, and third axes, respectively (Fig. 5). The first three principal components explained $59.1 \%$ of all the variation among the isolates. Eigenvectors of the first, second, and third components (ascospores produced on corn stalks, total amount of trichothecenes in wheat kernels, and FHB severity in spikelets
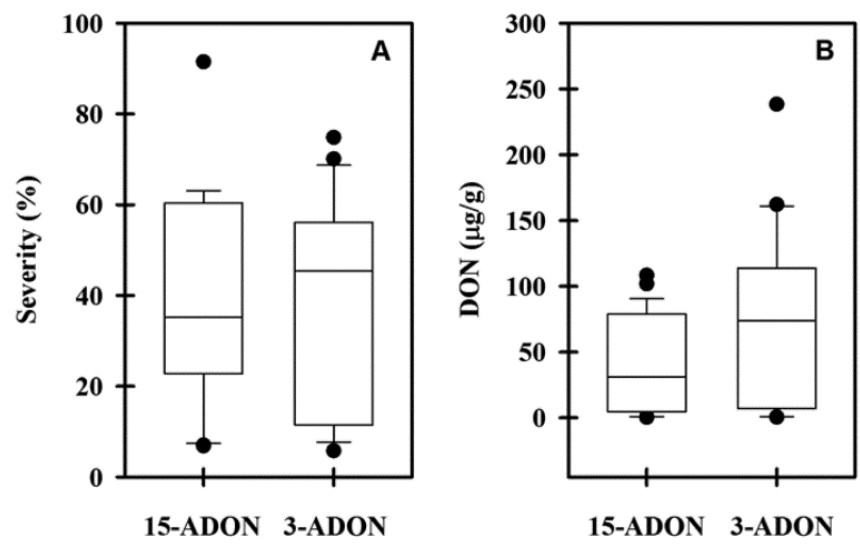

Fig. 3. Box plot and range of A, Fusarium head blight severity and B, deoxynivalenol (DON) among 25 Fusarium graminearum isolates possessing a 15-acetyl-deoxynivalenol (15-ADON) or 3-ADON genotype and inoculated onto 'Norm' wheat plants using the single-floret method. The line within the box represents the median, whereas the top and bottom lines of the boxes represent the 75th and 25th percentiles of the data, respectively. Vertical bars extending beyond the boxes represent the 10th and 90th percentiles and black circles indicate outliers.
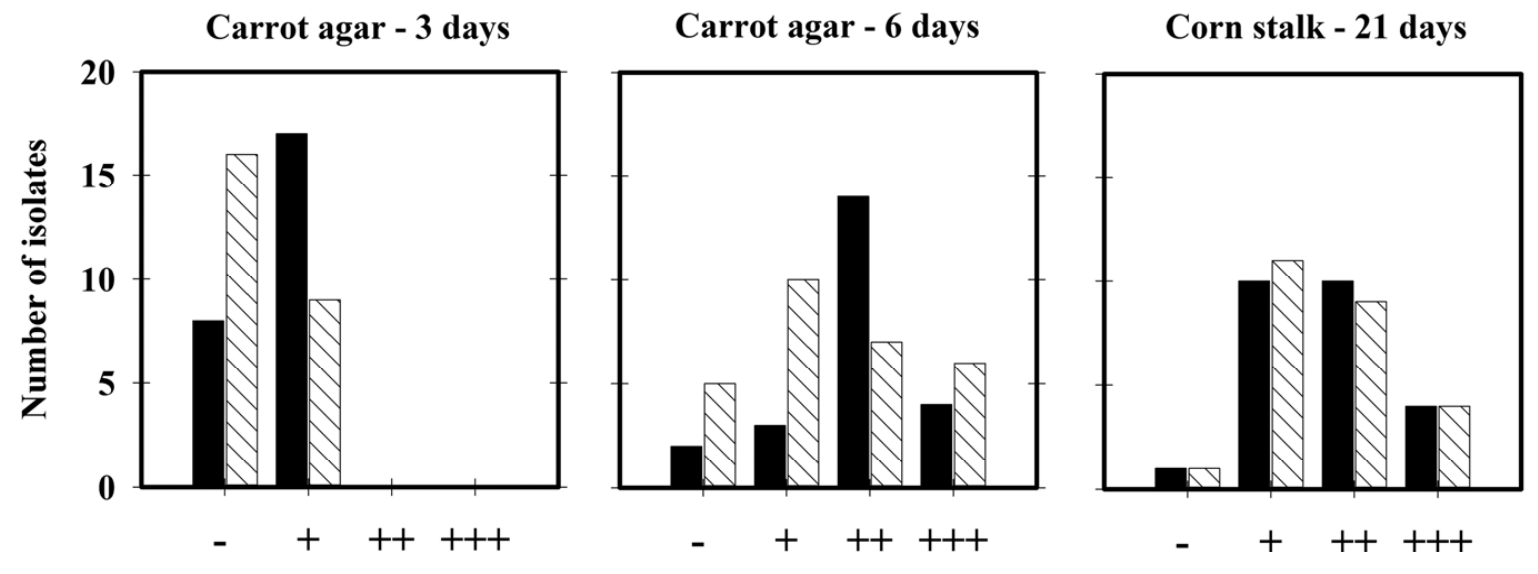

Perithecia formation

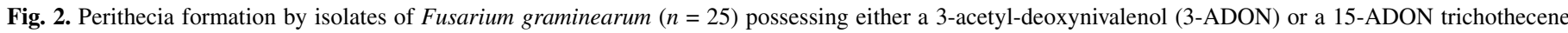

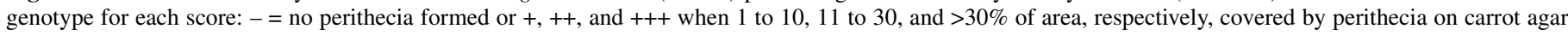

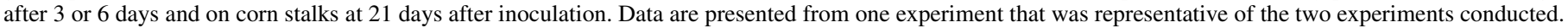


up of the point of inoculation) explained 29.4, 18.9, and $10.8 \%$ of the variation among the isolates, respectively. In the cluster analysis, the bootstrapping procedure grouped the isolates into two sharp groups ( $P \geq 0.350$ ), with 27 and 23 isolates each (Fig. 5). Altogether, the analyses showed that individuals of the two genotypes were present in both groups and in similar proportion (15-ADON/3-ADON = 14/13 and 11/12) (Fig. 5). The same was valid when the individuals were classified based on the true chemotypes determined by chemical analysis (15-ADON/ $3-\mathrm{ADON}=13 / 14$ and 12/11).

\section{DISCUSSION}

In this study, populations of 3-ADON and 15-ADON genotypes could not be differentiated for most of the measures of saprophytic and pathogenic fitness. Ascospore production on carrot agar was greater among 3-ADON isolates but this was not true for ascospore production on corn stalks, a natural substrate. Mycelial growth at any of the temperatures tested and the temperature sensitivity did not differ significantly between the 3-ADON and $15-\mathrm{ADON}$ isolates. The temperature sensitivity measure has been proposed as a surrogate for thermal adaptation (58) and saprophytic fitness (54). Yearly fluctuation in the frequencies of $3-\mathrm{ADON}$ and 15-ADON has been suggested to be associated with variability in seasonal temperatures (22). Using a mixture of 3-ADON/15-ADON (1:1) inoculum to spray inoculate wheat, a significantly higher frequency of 3-ADON isolates was recovered from plants incubated at 28 than at $20^{\circ} \mathrm{C}$ (22). Likewise, a significantly greater amount of DNA from 3-ADON isolates was found in barley previously inoculated with a mix of 15- and 3 -ADON at higher temperatures (8). However, our results did not show a clear difference $(P=0.068)$ in temperature sensitivity, as defined by the ratio of growth at 30 and $15^{\circ} \mathrm{C}$, between the $3-\mathrm{ADON}$ and $15-\mathrm{ADON}$ isolates. More studies are necessary to test the hypothesis that 3 -ADON isolates could have an adaptive advantage at higher temperatures.

The 15-ADON isolates formed perithecia on carrot agar faster than the 3-ADON isolates but large variability among the isolates was found in regard to the intrinsic ability to form perithecia and ascospores, similar to previous reports $(7,19)$. Differences in the duration of perithecia maturation was considered a source of error in measurements of ascospore production (19), which may explain the difference in this trait between the two trichothecene genotypes in our study. However, such difference was not found when using corn stalks as a substrate. Despite the fact that carrot agar media has been traditionally used in studies of $F$. graminearum sexual fertility $(15,19,52,53)$, perithecia formed on corn stalks, a common source of inoculum for epidemics, may lead to more epidemiologically relevant results $(13,16,27,50)$.

The aggressiveness of $F$. graminearum isolates has been positively correlated with their DON-producing ability (32). The isolates that we studied varied widely in trichothecene production and aggressiveness on one susceptible wheat variety but they could not be differentiated in these traits by trichothecene genotype, similar to the findings of Foroud et al. (17). Similarly, Purahong et al. (43) reported a wide range of aggressiveness levels among $32 \mathrm{~F}$. graminearum isolates isolated from durum wheat cultivated in northern-central Italy and no significant difference in aggressiveness was observed among the three different chemotypes (15-ADON, 3-ADON, and NIV).

In our study, nine isolates among the 12 highest DON prducers were of the 3-ADON genotype. However, despite a trend in which

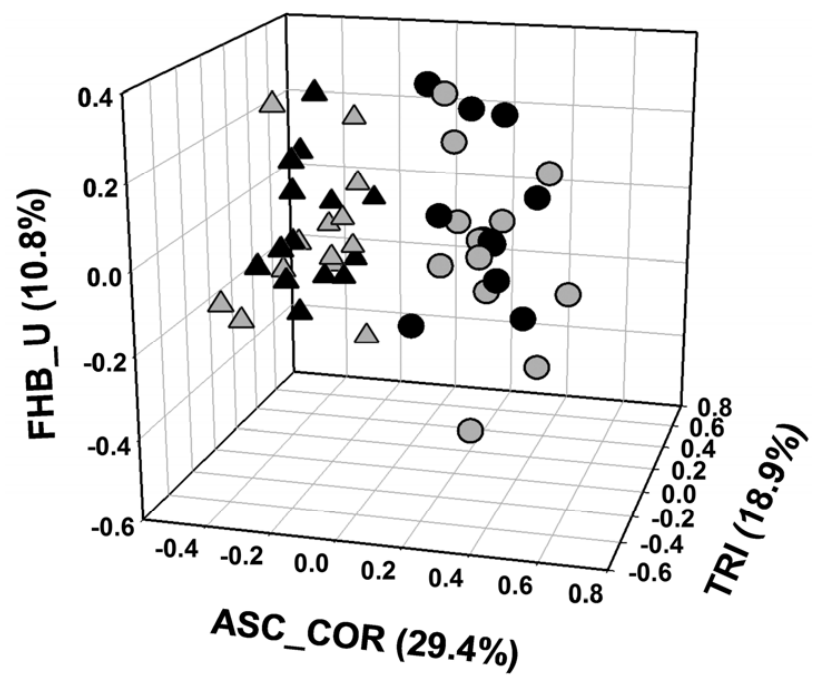

Fig. 5. Ordination of Fusarium graminearum isolates. The method is principal components analysis. Labels identify groups found in cluster analysis. First component: ASC_COR, counting of CFU discharged on agar media from inoculated corn stalks; second component: TRI, deoxynivalenol (DON) + 15acetyl-deoxynivalenol (15-ADON) + 3ADON; third component: FHB_U, incidence of symptomatic spikes up from the point of inoculation (central spikelet) on 'Norm' plants. Circle $=$ group 1 , triangle $=$ group 2 , black $=$ 15 -ADON isolates, and gray $=3$-ADON isolates.

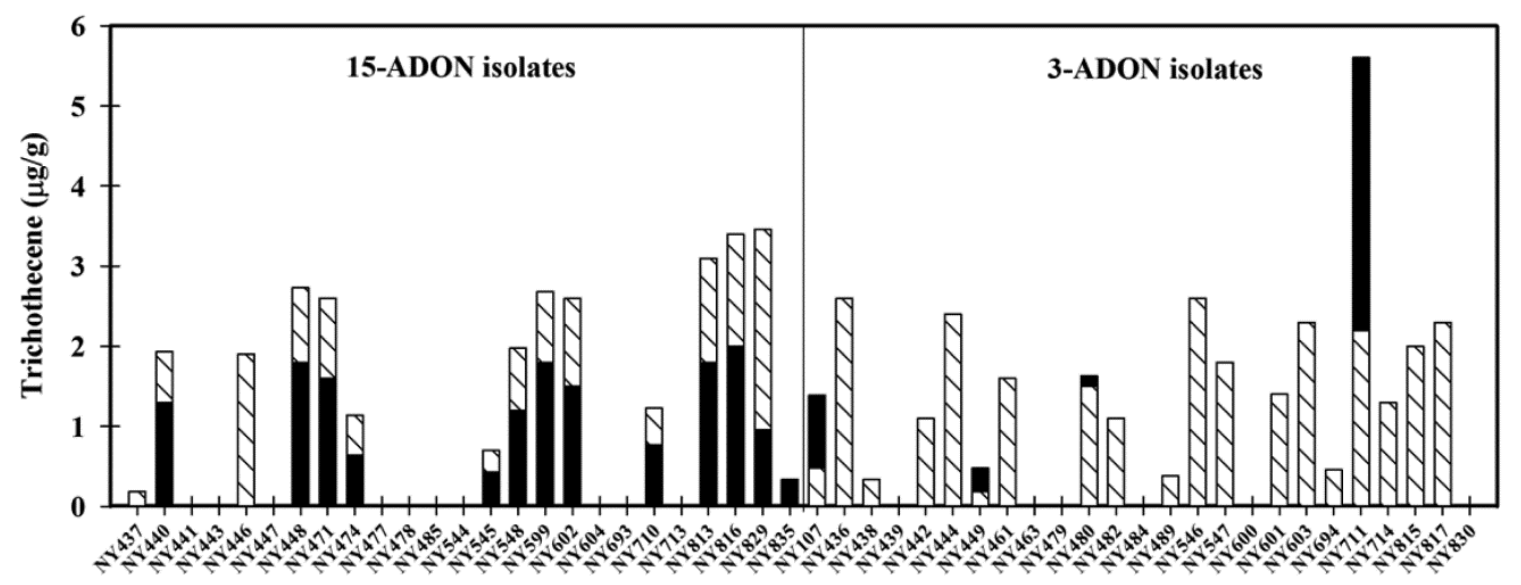

Fusarium graminearum isolates $(\mathbf{n}=\mathbf{5 0})$

15-acetyldeoxynivalenol $\quad \backslash \nabla$ 3-acetyldeoxynivalenol

Fig. 4. Mean production of 15-acetyl-deoxynivalenol (15-ADON) and 3-ADON by 25 Fusarium graminearum isolates of each trichothecene genotype. 
3-ADON isolates induced higher levels of DON than 15-ADON isolates, no significant difference was observed between the two trichothecene groups. A similar report was made by Gale et al. (20) in Minnesota.

As expected, 15-ADON and 3-ADON isolates produced more of the corresponding acetylate form than the other trichothecene genotype group. However, some isolates of the same trichothecene genotype group were able also to produce the acetylate form, in inoculated kernels, expected for the other group. This was more evident for 15-ADON genotypes, which produced detectable amounts of the 3-ADON mycotoxin, in some cases equal to or higher than the 15-ADON mycotoxin, thus demonstrating that the actual chemotype of that isolate would be 3-ADON. Previous studies to elucidate the genetic basis of the production of these two DON acetylates by $F$. graminearum isolates revealed that 3ADON production in solid substrate by 15 -ADON genotypes seems to be under regulation of the Tril01 gene and not the Tri3 gene, which seemed to regulate production of 15-ADON mycotoxin by $3-\mathrm{ADON}$ genotypes, especially due to $\mathrm{DON}$ acetylation by Tri3 (2,21). Because DON is a poor substrate for the action of Tri3, only trace amounts of $15-\mathrm{ADON}$ should be expected in solid substrate inoculated with 3-ADON isolates (2), which was the case in our study. Similar discrepancies between genotype and phenotype with respect to mycotoxin production potential of F. graminearum were observed by Mugrabi de Kuppler et al. (34), where one "molecular chemotype" (here, the trichothecene genotype) was able to produce mycotoxins characteristic of the other chemotype in considerable amount. With the widespread use of molecular genotyping to estimate the trichothecene phenotype proportions of regional populations of $F$. graminearum sensu lata, further research is needed to understand discrepancies between genotype and true chemotype identification, and perhaps more precise molecular markers for chemotype can be developed.

Several fitness measures were significantly correlated, with some of these being expected based on the fact that they derive one from another or they have been documented by previous evidence. All measures of pathogenic traits were highly correlated with the total amount of trichothecene in wheat kernels, especially disease severity, which could be associated with the role of DON as a virulence factor $(26,42)$ and with the fact that wheat spikes with more severe disease symptoms would be likely to have greater colonization and fungal biomass, thus leading to higher DON levels. FDK was highly correlated with DON concentration in wheat kernels, as demonstrated previously $(9,37)$.

Among the measurements of saprophytic fitness, the significant correlation of mycelial growth on PDA with the production of ascospores on corn stalks provides further evidence that corn stalks are a more relevant choice than carrot agar for studies of pathogen biology and FHB epidemiology (27).

The multivariate analyses used, to our knowledge, for the first time to determine overall fitness of 3- and 15-ADON F. graminearum isolates did not provide evidence of a difference between the two populations because the two sharp clusters were composed of isolates of both 3- and 15-ADON genotypes. Previously, Tunali et al. (54) used PCA to assess the overall fitness of 30 isolates of three species (F. culmorum, F. graminearum, and $F$. pseudograminearum) associated with head blight and crown rot in Australia and based on 13 saprophytic and pathogenic traits; these species could not be differentiated and no information about the acetylate forms produced by the $10 \mathrm{~F}$. graminearum isolates was provided. Also, Talas et al. (51), studying genetic diversity of 90 isolates of $F$. graminearum from three distinct wheat fields (30 isolates/field), delineated four distinct groups but, again, no information was provided on the trichothecene genotypes of the isolates.

According to Ward et al. (57), there is a contribution to the $15-A D O N$ genome from 3-ADON isolates. Sexual crossing between and within chemotypes may occur in the field (7) and under laboratory conditions (19). One could speculate that early immigrant isolates of the 3-ADON genotype may have possessed genes conferring fitness superior to those of antecedent 15-ADON genotypes in the region. However, through generations of recombination and natural selection, those introduced genes may now be present in isolates of both trichothecene genotypes. The proportion of trichothecene genotypes of 85\% 15-ADON/15\% 3-ADON found in a survey of western New York wheat in 2007 (47) is identical to the proportion found in a survey done in western New York in 2011 (G. C. Bergstrom, unpublished). There appears to be no significant ongoing shift for trichothecene genotype in contemporary populations of $F$. graminearum on wheat in western New York and that is consistent with the results reported in this article, showing that there is no clear distinction in fitness attributes between isolates of the 3-ADON and 15-ADON genotypes. However, the very great variation in specific attributes of fitness within this regional population of $F$. graminearum suggests that further research is needed to understand how these traits may be related to fungal life history and how they might lead to new insights to be exploited for improved disease management.

\section{ACKNOWLEDGMENTS}

We thank the Programa de Pós-graduação em Fitotecnia (UFRGS) and CNPq-Conselho Nacional de desenvolvimento Científico e Tecnológico for providing a graduate scholarship and a sandwich fellowship to P. Spolti, and the CNPq for providing a research fellowship to E. M. Del Ponte. Laboratory, greenhouse, and field expenditures for this research were provided by Cornell University Hatch Project NYC153473.

\section{LITERATURE CITED}

1. Akinsanmi, O. A., Chakraborty, S., Backhouse, D., and Simpfendorfer, S. 2007. Passage through alternative hosts changes the fitness of Fusarium graminearum and Fusarium pseudograminearum. Environ. Microbiol. 9:512-520

2. Alexander, N. J., McCormick, S. P., Waalwijk, C., van der Lee, T., and Proctor, R. H. 2011. The genetic basis for 3-ADON and 15-ADON trichothecene chemotypes in Fusarium. Fungal Genet. Biol. 48:485-495.

3. Astolfi, P., dos Santos, J., Schneider, L., Gomes, L. B., Silva, C. N., Tessmann, D. J., and Del Ponte, E. M. 2011. Molecular survey of trichothecene genotypes of Fusarium graminearum species complex from barley in southern Brazil. Int. J. Food Microbiol. 148:197-201.

4. Burlakoti, R. R., Neate, S. M., Adhikari, T. B., Gyawali, S., Salas, B., Steffenson, B. J., and Schwarz, P. B. 2011. Trichothecene profiling and population genetic analysis of Gibberella zeae from barley in North Dakota and Minnesota. Phytopathology 101:687-695.

5. Cavinder, B., Sikhakolli, U., Fellows, K. M., and Trail, F. 2012. Sexual development and ascospore discharge in Fusarium graminearum. J. Vis. Exp. Online publication. doi:10.3791/3895

6. Chandler, E. A., Simpson, D. R., Thomsett, M. A., and Nicholson, P. 2003. Development of PCR assays to Tri7 and Tril3 trichothecene biosynthetic genes, and characterisation of chemotypes of Fusarium graminearum, Fusarium culmorum and Fusarium cerealis. Physiol. Mol. Plant Pathol. 62:355-367.

7. Chen, Y., and Zhou, M.-G. 2009. Characterization of Fusarium graminearum isolates resistant to both carbendazim and a new fungicide JS399-19. Phytopathology 99:441-446.

8. Clear, R. M., Tucker, J. R., Gaba, D., Patrick, S. K., Lee, S.-J., Demeke, T., Tittlemier, S. A., Legge, W. G., and Gräfenhan, T. 2013. Deoxynivalenol levels and chemotype frequency in barley cultivars inoculated with two chemotypes of Fusarium graminearum. Can. J. Plant Pathol. 35:37-45.

9. Cowger, C., and Arellano, C. 2010. Plump kernels with high deoxynivalenol linked to late Gibberella zeae infection and marginal disease conditions in winter wheat. Phytopathology 100:719-728.

10. Crane, J. M., Gibson, D. M., Vaughan, R. H., and Bergstrom, G. C. 2013. Iturin levels on wheat spikes linked to biological control of Fusarium head blight by Bacillus amyloliquefaciens. Phytopathology 103:146-155.

11. Del Ponte, E. M., Fernandes, J. M. C., and Bergstrom, G. C. 2007. Influence of growth stage on Fusarium head blight and deoxynivalenol production in wheat. J. Phytopathol. 155:577-581.

12. Desjardins, A., 2006. Trichothecenes. Pages 13-64 in: Fusarium Mycotoxins: Chemistry, Genetics, and Biology. A. Desjardins, ed. The American Phytopathological Society, St. Paul, MN. 
13. Dill-Macky, R., and Jones, R. K. 2000. The effect of previous crop residues and tillage on Fusarium head blight of wheat. Plant Dis. 84:71-76.

14. Eudes, F., Comeau, A., Rioux, S., and Collin, J. 2001. Impact of trichothecenes on Fusarium head blight [Fusarium graminearum] development in spring wheat (Triticum aestivum). Can. J. Plant Pathol. 23:318-322.

15. Fan, J., Urban, M., Parker, J. E., Brewer, H. C., Kelly, S. L., HammondKosack, K. E., Fraaije, B. A., Liu, X., and Cools, H. J. 2013. Characterization of the sterol 14a-demethylases of Fusarium graminearum identifies a novel genus-specific CYP51 function. New Phytol. Online publication. doi: $10.1111 / \mathrm{nph} .12193$

16. Fernando, W. G. D., Paulitz, T. C., Seaman, W. L., Dutilleul, P., and Miller, J. D. 1997. Head blight gradients caused by Gibberella zeae from area sources of inoculum in wheat plots. Phytopathology 87:414-421.

17. Foroud, N. A., McCormick, S. P., MacMillan, T., Badea, A., Kendra, D. F., Ellis, B. E., and Eudes, F. 2012. Greenhouse studies reveal increased aggressiveness of emergent Canadian Fusarium graminearum chemotypes in wheat. Plant Dis. 96:1271-1279.

18. Fuentes, R. G., Mickelson, H. R., Busch, R. H., Dill-Macky, R., Evans, C. K., Thompson, W. G., Wiersma, J. V., Xie, W., Dong, Y., and Anderson, J. A. 2005. Resource allocation and cultivar stability in breeding for Fusarium head blight resistance in spring wheat. Crop Sci. 45:1965-1972.

19. Fuentes-Bueno, I. 2012. Dissection of fertility barriers among lineages of Gibberella zeae. M.Sc. thesis, Kansas State University.

20. Gale, L. R., Dill-Macky, R., Anderson, J. A., Smith, K. P., and Kistler, H. C. 2009. Aggressiveness and mycotoxin potential of U.S. Fusarium graminearum populations in field-grown wheat and barley. Page 173 in: Proc. 2009 Natl. Fusarium Head Blight Forum. S. Canty, A. Clark, J. Mundell, E. Walton, D. Ellis, and D. Van Sanford, eds. Orlando, FL.

21. Garvey, G. S., McCormick, S. P., Alexander, N. J., and Rayment, I. 2009. Structural and functional characterization of TRI3 trichothecene 15-Oacetyltransferase from Fusarium sporotrichioides. Protein Sci. 18:747761.

22. Gilbert, J., Clear, R., Patrick, S., Slusarenko, K., and Wolfe, C. 2012. Ratio of 3-ADON and 15-ADON isolates of Fusarium graminearum recovered from wheat plants inoculated and incubated at various temperatures and from field nurseries. Can. J. Plant Pathol. 34:112.

23. Gilbert, J., and Haber, S. 2013. Overview of some recent research developments in Fusarium head blight of wheat. Can. J. Plant Pathol. 35:149-174.

24. Goswami, R. S., and Kistler, H. C. 2005. Pathogenicity and in planta mycotoxin accumulation among members of the Fusarium graminearum species complex on wheat and rice. Phytopathology 95:1397-1404.

25. Guo, X. W., Fernando, W. G. D., and Seow-Brock, H. Y. 2008 Population structure, chemotype diversity, and potential chemotype shifting of Fusarium graminearum in wheat fields of Manitoba. Plant Dis. 92:756762.

26. Ilgen, P., Hadeler, P., Maier, F. J., and Schäfer, W. 2009. Developing kernel and rachis node induce the trichothecene pathway of Fusarium graminearum during wheat head infection. Mol. Plant-Microbe Interact. 22:899-908.

27. Keller, M. D., Waxman, K. D., Bergstrom, G. C., and Schmale, D. G., III. 2010. Local distance of wheat spike infection by released clones of Gibberella zeae disseminated from infested corn residue. Plant Dis. 94:1151-1155.

28. Klittich, C. J. R., and Leslie, J. F. 1988. Nitrate reduction mutants of Fusarium moniliforme (Gibberella fujikuoi). Genetics 118:417-423.

29. Leplat, J., Friberg, H., Abid, M., and Steinberg, C. 2013. Survival of Fusarium graminearum, the causal agent of Fusarium head blight. A review. Agron. Sustain. Dev. 33:97-111

30. Maldonado-Ramirez, S. L. 2001. Aerobiology of the wheat scab fungus, Gibberella zeae: Discharge, atmospheric dispersal, and deposition of ascospores. Ph.D. dissertation, Cornell University, Ithaca, NY.

31. McMullen, M., Bergstrom, G. C., De Wolf, E., Dill-Macky, R., Hershman, D., Shaner, G., and Van Sanford, D. V. 2012. A unified effort to fight an enemy of wheat and barley: Fusarium head blight. Plant Dis. 96:1712-1728.

32. Mesterházy, A. 2002. Role of deoxynivalenol in aggressiveness of Fusarium graminearum and $F$. culmorum and in resistance to Fusarium head blight. Eur. J. Plant Pathol. 108:675-684.

33. Mirocha, C. J., Kolaczkowski, E., Xie, W., Yu, H., and Jelen, H. 1998. Analysis of deoxynivalenol and its derivatives (batch and single kernel) using gas chromatography/mass spectrometry. J. Agric. Food Chem. 46:1414-1418.

34. Mugrabi de Kuppler, A. L., Steiner, U., Sulyok, M., Krska, R., and Oerke, E. C. 2011. Genotyping and phenotyping of Fusarium graminearum isolates from Germany related to their mycotoxin biosynthesis. Int. J. Food Microbiol. 151:78-86.

35. Nielsen, L. K., Jensen, J. D., Rodríguez, A., Jørgensen, L. N., and Justesen, A. F. 2012. TRI12 based quantitative real-time PCR assays reveal the distribution of trichothecene genotypes of $F$. graminearum and
F. culmorum isolates in Danish small grain cereals. Int. J. Food Microbiol. 157:384-392.

36. Pan, D., Calero, N., Mionetto, A., and Bettucci, L. 2013. Trichothecene genotypes of Fusarium graminearum from wheat in Uruguay. Int. J. Food Microbiol. 162:120-123.

37. Paul, P. A., Lipps, P. E., and Madden, L. V. 2005. Relationship between visual estimates of Fusarium head blight intensity and deoxynivalenol accumulation in harvested wheat grain: a meta-analysis. Phytopathology 95:1225-1236

38. Pillar, V. D. 1999. How sharp are classifications? Ecology 80:2508-2516.

39. Pillar, V. D. 1999. The bootstrapped ordination reexamined. J. Veg. Sci. 10:895-902.

40. Pillar, V. D. 2006. MULTIV sofware para análise multivariada, testes de aleatorização e autoreamostragem "bootstrap", v.2.4.2. Departamento de Ecologia, Universidade Federal do Rio Grande do Sul, Porto Alegre, Brazil.

41. Pillar, V. D., and Orlóci, L. 1996. On randomization testing in vegetation science: multifactor comparisons of relevé groups. J. Veg. Sci. 7:582-592.

42. Proctor, R. H., Hohn, T. M., and McCormick, S. P. 1995. Reduced virulence of Gibberella zeae caused by disruption of a trichothecene toxin biosynthetic gene. Mol. Plant-Microbe Interact. 8:593-601.

43. Purahong, W., Nipoti, P., Pisi, A., Lemmens, L., and Prodi, A. 2013 Aggressiveness of different Fusarium graminearum chemotypes within a population from Northern-Central Italy. Mycoscience. Online publication. http://dx.doi.org/10.1016/j.myc.2013.05.007

44. Puri, K. D., and Zhong, S. 2010. The 3ADON population of Fusarium graminearum found in North Dakota is more aggressive and produces a higher level of DON than the prevalent 15ADON population in spring wheat. Phytopathology 100:1007-1014.

45. Reynoso, M. M., Ramirez, M. L., Torres, A. M., and Chulze, S. N. 2011. Trichothecene genotypes and chemotypes in Fusarium graminearum strains isolated from wheat in Argentina. Int. J. Food Microbiol. 28:444-448.

46. Sarver, B. J., Ward, T. J., Gale, L. R., Broz, K., Kistler, H. C., Aoki, T., Nicholson, P., Carter, J., and O’Donnell, K. 2011. Novel Fusarium head blight pathogens from Nepal and Louisiana revealed by multilocus genealogical concordance. Fungal Genet. Biol. 48:1096-107.

47. Schmale, D. G., Wood-Jones, A. K., Cowger, C., Bergstrom, G. C., and Arellano, C. 2011. Trichothecene genotypes of Gibberella zeae from winter wheat fields in the eastern USA. Plant Pathol. 60:909-917.

48. Spolti, P., Del Ponte, E. M., and Bergstrom, G. C. 2012. The 3-ADON and 15-ADON genotypes of Fusarium graminearum in New York are not discriminated by pathogenic or sexual reproductive fitness. Page 124 in: Proc. 2012 Natl. Fusarium Head Blight Forum. S. Canty, A. Clark, A. Anderson-Scully, and D. Van Sanford, eds. U.S. Wheat \& Barley Scab Research Initiative, East Lansing, MI/Lexington, KY.

49. Starkey, D. E., Ward, T. J., Aoki, T., Gale, L. R., Kistler, H. C., Geiser, D. M., Suga, H., Tóth, B., Varga, J., and O’Donnell, K. 2007. Global molecular surveillance reveals novel Fusarium head blight species and trichothecene diversity. Fungal Genet. Biol. 44:1191-1204.

50. Sutton, J. C. 1982. Epidemiology of wheat head blight and maize ear rot caused by Fusarium graminearum. Can. J. Plant Pathol. 4:195-209.

51. Talas, F., Kalih, R., and Miedaner, T. 2012. Within-field variation of Fusarium graminearum isolates for aggressiveness and deoxynivalenol production in wheat head blight. Phytopathology 102:128-134.

52. Trail, F., and Common, R. 2000. Perithecial development by Gibberella zeae: a light microscopy study. Mycologia 92:130-138.

53. Trail, F., Xu, H., and Loranger, R. 2002. Physiological and environmental aspects of ascospore discharge in Gibberella zeae (anamorph Fusarium graminearum). Mycologia 94:181-189.

54. Tunali, B., Obanor, F., Erginbaș, G., Westecott, R. A., Nicol, J., and Chakraborty, S. 2012. Fitness of three Fusarium pathogens of wheat. FEMS Microbiol. Ecol. 81:596-609.

55. Von der Ohe, C., Gauthier, V., Tamburic-Ilincic, L., Brule-Babel, A., Fernando, W. G. D., Clear, R., Ward, T. J., and Miedaner, T. 2010. A comparison of aggressiveness and deoxynivalenol production between Canadian Fusarium graminearum isolates with 3-acetyl and 15-acetyldeoxynivalenol chemotypes in field-grown spring wheat. Eur. J. Plant Pathol. 127:407-417.

56. Ward, T. J., Bielawski, J. P., Kistler, H. C., Sullivan, E., and O'Donnell, K. 2002. Ancestral polymorphisms and adaptive evolution in the tricothecene mycotoxin gene cluster of phytopathogenic Fusarium. Proc. Natl. Acad. Sci. USA 99:9278-9283.

57. Ward, T. J., Clear, R. M., Rooney, A. P., O’Donnell, K., Gaba, D., Patrick, S., Starkey, D. E., Gilbert, J., Geiser, D. M., and Nowicki, T. W. 2008. An adaptive evolutionary shift in Fusarium head blight pathogen populations is driving the rapid spread of more toxigenic Fusarium graminearum in North America. Fungal Genet. Biol. 45:473-484.

58. Zhan, J., and McDonald, B. A. 2011. Thermal adaptation in the fungal pathogen Mycosphaerella graminicola. Mol. Ecol. 20:1689-1701. 